\title{
MicroRNA-323 suppresses nerve cell toxicity in cerebral infarction via the transforming growth factor-ß31/SMAD3 signaling pathway
}

\author{
FENGLI CHE, HUISHAN DU, JIANCHAO WEI, WEIDONG ZHANG, ZHE CHENG and YANNA TONG \\ Department of Neurology, Beijing Luhe Hospital Capital Medical University, Beijing 101145, P.R. China
}

Received December 11, 2017; Accepted October 19, 2018

DOI: 10.3892/ijmm.2018.4020

\begin{abstract}
In the present study, the aim was to investigate the function of microRNA-323 (miR-323) in cerebral infarction and its underlying mechanism. A rat model of cerebral infarction was established and hippocampal tissues were analyzed. In addition, to further understand the role of miR-323, PC12 cells were transfected with miR-323 mimics or inhibitors and subjected to hypoxia to model cerebral infarction. Reverse transcription-quantitative polymerase chain reaction was used to measure the expression of miR-323. A luciferase reporter assay was conducted to analyze miR-323 target sites the partial sequence of the 3'-untranslated region of SMAD3 mRNA in vitro. Western blot analysis was also used to analyze transforming growth factor- $\beta 1$ (TGF- $\beta 1$ ) and SMAD3 protein expression levels. It was observed that miR-323 expression was significantly upregulated in rats with cerebral infarction compared with rats in the sham-control group. In addition, overexpression of miR-323 induced nerve cell toxicity and reduced nerve cell growth in an in vitro model of cerebral infarction, whereas downregulation of miR-323 caused the opposite effects on nerve cell toxicity and growth in this model. In addition, overexpression of miR-323 directly targeted and suppressed SMAD3 expression in the in vitro model of cerebral infarction, while inhibition of miR-323 induced SMAD3 expression. The use of a SMAD3 inhibitor suppressed the effect of anti-miR-323 on nerve cell toxicity in the in vitro model of cerebral infarction. Collectively, these findings suggested that miR-323 suppresses nerve cell apoptosis in cerebral infarction via the TGF- $\beta 1 /$ SMAD3 signaling pathway.
\end{abstract}

\section{Introduction}

Acute cerebral infarction (ACI) is a common disease with high morbidity, disability rate and mortality worldwide, as well as

Correspondence to: Dr Fengli Che, Department of Neurology, Beijing Luhe Hospital Capital Medical University, 28 New South Road, Tongzhou, Beijing 101145, P.R. China

E-mail: chensh187@yeah.net

Key words: microRNA-323, transforming growth factor- $\beta 1$, SMAD3, cerebral infarction in China (1). It affects the quality of life of patients and adds to the economic burden on the society and the patient's family (1). ACI is a disease with etiological heterogeneity; therefore, extensively investigating blood biomarkers reflecting the ACI pathophysiological changes has great clinical significance and application value (2). These biomarkers can be used for early diagnosis, etiology recognition and selection of early clinical individualized treatment (2). In addition, they can predict disease prognosis, assisting in the search for novel targets for disease intervention $(2,3)$.

MicroRNAs (miRNAs or miRs) are a class of small endogenous non-coding single-stranded RNA molecules and one of the primary regulatory factors of gene expression (4). miRNA interacts with the specific sequence of its target gene to suppress the target gene activity or promote its degradation following transcription (5). Furthermore, it regulates target gene expression and participates in various biological processes, including cell proliferation, differentiation, apoptosis and metabolism. A brain-specific miRNA is abundant in the central nervous system (6); however, it is not expressed or is rarely expressed in other organs, and serves a vital role in neurogenesis and function (6). It is estimated that miRNAs may be passively leaked from broken tissue and cells or invasive cells, similar to other substances. Alternatively, they may be actively secreted into the blood circulation from the damaged tissue and cells (6). Previous animal and clinical research reported that miRNA expression profiles in the brain tissue and blood are aberrantly altered following an ischemic stroke (3). Such findings indicate that stroke-associated miRNAs are involved in the pathophysiological process occurring subsequent to cerebral ischemia (7).

The transforming growth factor- $\beta$ (TGF- $\beta$ )/Smad signaling transduction pathway is involved in the genesis and development of Alzheimer's disease, hydrocephalus and neuroglioma (8). It also participates in the pathological process of rat cerebral ischemia reperfusion injury (8). The TGF- $\beta$ level has been reported to be markedly higher in patients with ACI (8). This suggests that TGF- $\beta$ participates in the pathogenesis of ACI and is an important reference molecule for diagnosing ACI recurrence (8). Smad proteins also participate in multiple pathological processes, such as cerebral injury. Research has been conducted to analyze cortical neuron morphological changes caused by experimental communicating hydrocephalus, as well as the effect of the TGF- $\beta /$ Smad signaling pathway on these changes (8). The results indicate that abnormal Smad 
protein expression is involved in the neuron damage process occurring in hydrocephalus (8). Therefore, the present study aimed to examine the function of miR-323 in ACI and its underlying mechanism.

\section{Materials and methods}

Animals and ACI model establishment. Male adult Sprague-Dawley rats (weight, 220-250 g; $\mathrm{n}=12$ ) were purchased from Beijing Vital River Laboratory Animal Technology Co., Ltd. (Beijing, China). All rats were housed at $22-23^{\circ} \mathrm{C}$ at $55 \%$ humidity and a 12/12 h light/dark cycle. Food and water were available ad libitum. The rats were randomly divided into the sham surgery group (serving as the control) and the ACI model group ( $\mathrm{n}=6$ rats per group). The animals were anesthetized by pentobarbital sodium $(30 \mathrm{ml} / \mathrm{kg}$; intraperitoneal injection), and then a surgical midline incision was made to expose the internal carotid artery, external carotid artery and right common carotid artery. A nylon suture was inserted into the right common carotid artery and injected at $18 \mathrm{~mm}$ into the internal carotid artery. After $2 \mathrm{~h}$ of the injection, the nylon sutures were gently removed from the internal carotid artery and reperfusion was performed for $1 \mathrm{~h}$. Rats in the control group received the same surgical procedures without the insertion of the monofilament nylon suture. After $24 \mathrm{~h}$, the animals were anesthetized by pentobarbital sodium $(30 \mathrm{ml} / \mathrm{kg}$; intraperitoneal injection), sacrificed and hippocampal tissues were extracted. The animal experiments were approved by the Ethics Committee of the Beijing Luhe Hospital Capital Medical University (Beijing, China).

$H \& E$ staining. Hippocampal tissues were fixed with $4 \%$ paraformaldehyde for $24 \mathrm{~h}$ at room temperature. Following this, tissues were dehydrated using ethanol, embedded in paraffin and then cut into sections $(10 \mu \mathrm{m})$. Sections were then stained with $\mathrm{H} \& \mathrm{E}$ for $10 \mathrm{~min}$ at room temperature and observed using a light microscope (magnification, x100; Leica Microsystems $\mathrm{GmbH}$, Wetzlar, Germany) and analyzed using Image Lab 3.0 (Bio-Rad Laboratories, Inc., Hercules, CA, USA).

Gene microarray. Total protein was extracted using a radioimmunoprecipitation assay lysis buffer (Beyotime Institute of Biotechnology, Nanjing, China) and then protein concentration was determined using a bicinchoninic acid assay (Beyotime Institute of Biotechnology). Total RNA (500 ng) obtained from hippocampal tissues or transfected cells was hybridized to the SurePrint G3 Mouse Whole Genome GE 8 X60 K Microarray G4852A platform (G4471A-021828; Agilent Technologies, Inc., Santa Clara, CA, USA), to analyze signaling proteins, including IL-1 $\beta$, IL-6 and PI3K. Images were quantified using Agilent Feature Extraction software (version A.10.7.3.1; Agilent Technologies, Inc.).

Cell culture and miRNA transfection. PC12 cells were purchased from the Institute of Biochemistry and Cell Biology (Shanghai, China). The cells were incubated in Dulbecco's modified Eagle's medium (DMEM; Hyclone; GE Healthcare Life Sciences, Beijing, China) with $10 \%$ fetal bovine serum (Gibco; Thermo Fisher Scientific, Inc., Waltham, MA, USA), $2 \mathrm{mmol} / \mathrm{l}$ glutamine (Invitrogen; Thermo Fisher Scientific,Inc.),
200 U/ml penicillin (Hyclone; GE Healthcare Life Sciences) and $100 \mu \mathrm{g} / \mathrm{ml}$ streptomycin (Hyclone; GE Healthcare Life Sciences) at $37^{\circ} \mathrm{C}$ with $5 \% \mathrm{CO}_{2}$.

miR-323, anti-miR-323 and control mimics were purchased from RiboBio Co., Ltd. (Guangzhou, China), and their sequences were as follows: miR-323, 5'-AUGUAAUGAGAC GGUUCUUUUUU-3'; anti-miR-323, 5'-AAAAAAGAACCG UCUCAUUACAU-3'; and control, 5'-AAUAGCAUCGAA AAGUCCGG-3'. A total of $100 \mathrm{nM}$ mimics were transfected into the cells using Invitrogen Lipofectamine ${ }^{\circledR} 3000$ (Thermo Fisher Scientific, Inc.), according to the manufacturer's protocol. The medium was changed after $4 \mathrm{~h}$ of transfection in $5 \% \mathrm{CO}_{2}$ incubator at $37^{\circ} \mathrm{C}$, and the cells were then incubated for 24,48 and $72 \mathrm{~h}$ time intervals. Following this, fresh DMEM was added into the cell culture without glucose under hypoxic conditions $\left(5 \% \mathrm{CO}_{2}\right.$ and $\left.95 \% \mathrm{~N}_{2}\right)$ and incubated for $6 \mathrm{~h}$. Following transfection at $37^{\circ} \mathrm{C}$, SMAD3 inhibitor $(1 \mu \mathrm{M}$ SIS3; MedChemExpress, Monmouth Junction, NJ, USA) or TGF- $\beta 1$ inhibitor (20 nm, LY2109761) was added to the cells and subsequently incubated for $48 \mathrm{~h}$ without glucose under hypoxic conditions $\left(5 \% \mathrm{CO}_{2}\right.$ and $\left.95 \% \mathrm{~N}_{2}\right)$.

Reverse transcription-quantitative polymerase chain reaction $(R T-q P C R)$. Total RNA samples were extracted from the transfected PC12 cells using TRIzol reagent (Invitrogen; Thermo Fisher Scientific, Inc.). RNA concentrations were determined using a microplate reader (Benchmark Plus; Bio-Rad Laboratories, Inc.) at a wavelength of $260 / 280 \mathrm{~nm}$. For the miRNA expression assay, RT was performed using a TaqMan miRNA assay kit (Applied Biosystems; Thermo Fisher Scientific, Inc.). Next, the PCR reaction was performed using a Bio-Rad iCycler iQ RealTime PCR detection system (Bio-Rad Laboratories, Inc.) and a $\mathrm{SYBR}^{\circledR}$ Premix Ex Taq ${ }^{\mathrm{TM}}$ II kit (Takara Biotechnology Co., Ltd., Dalian, China). The thermal cycling conditions included denaturation at $95^{\circ} \mathrm{C}$ for $15 \mathrm{~min}$, followed by 40 cycles of $95^{\circ} \mathrm{C}$ for $30 \mathrm{sec}$ and $60^{\circ} \mathrm{C}$ for $15 \mathrm{sec}$. The following primers were used in qPCR: miR-323 sense, 5'-GCGCCCCAGGAGGCTGATGC-3', and antisense, 5'-CGT GGTGGTCCCGCCGCC3-3'; and U6 sense, 5'-GTTTTGTAG TTTTTGGAGTTAGTGTTGTGT-3' and antisense, 5'-CTC AACCTACAATCAAAAACAACACAAACA-3'. miR-323 expression was normalized using the $2^{-\triangle \Delta C q}$ method (8).

Dual-luciferase reporter assay. The 3'-untranslated region (3'-UTR) of SMAD3 mRNA containing the predicted miR-323 binding sequences was amplified and subcloned into a pGL3 luciferase promoter vector (Invitrogen; Thermo Fisher Scientific, Inc.). pGL3 was then co-transfected with AAV-pre-miR-323 or controls into PC12 cells. Following incubation for $48 \mathrm{~h}$, the luciferase report activity was detected using the Dual-Luciferase Reporter Assay kit (Promega Corporation, Madison, WI, USA) according to the manufacturer's protocol.

Cell viability assay. The viability of each cell group at 24 , 48 and $72 \mathrm{~h}$ post-transfection was measured using an MTT

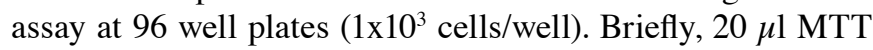
solution was added into the cells and incubated at $37^{\circ} \mathrm{C}$ for $4 \mathrm{~h}$. Dimethyl sulfoxide was then added for $20 \mathrm{~min}$ at $37^{\circ} \mathrm{C}$, and the absorbance was measured using a microplate reader 
(Benchmark Plus; Bio-Rad Laboratories, Inc.) at a wavelength of $490 \mathrm{~nm}$.

Lactate dehydrogenase $(\mathrm{LDH})$ activity. A total of $48 \mathrm{~h}$ post-transfection, the LDH activity of each cell group was measured using an LDH activity kit (Beyotime Institute of Biotechnology), according to the manufacturer's protocol. The absorbance was measured using a microplate reader (Benchmark Plus) at a wavelength of $450 \mathrm{~nm}$.

Flow cytometry. A total of $48 \mathrm{~h}$ post-transfection, the apoptosis rate of cells was measured using an FITC Annexin V kit (cat. no. 556420; BD Biosciences, San Jose, CA, USA). Briefly, cells were harvested, washed three times with PBS and resuspended with $150 \mu \mathrm{l}$ binding buffer. Next, cells were stained with $5 \mu \mathrm{l}$ FITC-conjugated Annexin V and $5 \mu \mathrm{l}$ PI for $15 \mathrm{~min}$ in the dark. The apoptosis rate was assessed by flow cytometry with a FACSCalibur and the CellQuest software (BD Biosciences).

Caspase-3/9 activity assay. Caspase-3 and caspase-9 activities were measured using the Caspase 3 Activity Assay kit (cat. no. C1115; Beyotime Institute of Biotechnology) or Caspase 9 Activity Assay kit (cat.no. C1158; Beyotime Institute of Biotechnology), according to the manufacturer's protocol. The absorbance was then measured using a microplate reader (Benchmark Plus) at a wavelength of $405 \mathrm{~nm}$.

Western blot analysis. Total protein in the cells was extracted using a radioimmunoprecipitation assay lysis buffer (Beyotime Institute of Biotechnology) and protein concentration was determined using a bicinchoninic acid assay (Beyotime Institute of Biotechnology). Protein (50 $\mu \mathrm{g})$ was then separated by $10 \%$ SDS-PAGE and blotted onto a pre-wet nitrocellulose membrane (GE Healthcare Life Sciences). Subsequent to blocking with $5 \%$ skimmed milk in TBST for $1 \mathrm{~h}$ at $37^{\circ} \mathrm{C}$, the membranes were immunoblotted with the following primary antibodies at $4^{\circ} \mathrm{C}$ overnight: SMAD2 (1:1,000; sc-8332), TGF- $\beta 1$ (1:1,000; sc-9043), SMAD3 (1:1,000; sc-8332), B-cell lymphoma 2-associated X protein (Bax; 1:1,000; sc-6236) and GAPDH (1:5,000; sc-25778; all purchased from Santa Cruz Biotechnology, Inc., Dallas, TX, USA). The membranes were then washed with TBST for 15 min at and immunoblotted with a secondary antibody conjugated to horseradish peroxidase (1:5,000; sc-2004 and sc-2005; Santa Cruz Biotechnology, Inc.) for $1 \mathrm{~h}$ at $37^{\circ} \mathrm{C}$. Immunolabeling was visualized using an enhanced chemiluminescence system (GE Healthcare Life Sciences) and analyzed using Image Lab 3.0 software (Bio-Rad Laboratories, Inc.).

Immunocytochemistry. Cell was washed with PBS and then fixed with $4 \%$ paraformaldehyde for $20 \mathrm{~min}$ at room temperature. Cells were then blocked with $5 \%$ bovine serum albumin (Nanjing Sunshine Biotechnology Co., Ltd., Nanjing, China) and $0.1 \%$ Tris-X100 in Tris-buffered saline containing $0.1 \%$ Tween-20 (TBST) for $1 \mathrm{~h}$ at room temperature. Following this, cells were incubated with SMAD2/3 antibodies (1:100; sc-8332; Santa Cruz Biotechnology, Inc.) at $4^{\circ} \mathrm{C}$ overnight and then washed with TBST for $15 \mathrm{~min}$ at room temperature. Cells were subsequently incubated with goat anti-rabbit IgG-CruzFluor ${ }^{\mathrm{TM}} 555$ (1:100; sc-362272; Santa Cruz Biotechnology, Inc.) for $1 \mathrm{~h}$ at $37^{\circ} \mathrm{C}$ and then washed with TBST for $15 \mathrm{~min}$ at room temperature. Cell was then stained with $10 \mu \mathrm{g} / \mathrm{ml}$ of 4',6-diamidino-2-phenylindole for 15 min in darkness at room temperature. Cells were subsequently analyzed at room temperature using a Zeiss Axioplan 2 confocal microscope (magnification, x200; Carl Zeiss AG, Oberkochen, Germany) and analyzed using Image Lab 3.0 (Bio-Rad Laboratories, Inc.).

Statistical analysis. Data are presented as the mean \pm standard deviation $(n=3)$ of at least three independent experiments using SPSS 17.0 (SPSS, Inc., Chicago, IL, USA). The difference between two independent groups was assessed using Student's t-test. The difference between three independent groups was assessed using one-way analysis of variance and Tukey's post hoc test. $\mathrm{P}<0.05$ was considered to indicate a statistically significant difference.

\section{Results}

miR-323 expression in rats with cerebral infarction. In the present study, cerebral infarction was established in rat hippocampal neurons, followed by analysis of miR-323 expression. As shown in Fig. 1A, a higher apoptotic rate was observed in the nerve cells of the ACI model group, as compared with that of the control group. As displayed in Fig. 1B and C, miR-323 expression was significantly upregulated in rats with cerebral infarction, compared with that in the sham-control group. These data suggested that miR-323 may participate in the nerve cell apoptosis in ACI.

miR-323 regulates nerve cell toxicity in the in vitro cell model. The function of miR-323 on nerve cell toxicity was investigated in the in vivo model of cerebral infarction in the present study. As shown in Fig. 2A, the expression level of miR-323 was significantly increased in the in vivo model following transfection with miR-323 mimic for $24 \mathrm{~h}$, compared with that in the control mimics group. Next, it was observed that overexpression of miR-323 significantly inhibited cell viability in the in vitro model and increased the LDH activity level, as compared with the negative-control mimics group (Fig. 2B-F). The expression level of miR-323 was then significantly decreased in the in vitro model by transfection with anti-miR-323 for $24 \mathrm{~h}$, compared with that in the group transfected with negative-control mimics (Fig. 3A). Downregulation of miR-323 significantly promoted cell viability and inhibited the $\mathrm{LDH}$ activity level in the in vitro model, in comparison with those in the negative-control mimics group (Fig. 3B-F). These results indicated that miR-323 promoted nerve cell toxicity in cells.

miR-323 regulates Bax/caspase signaling pathway in the in vivo model. To demonstrate the contribution of miR-323 in cerebral infarction-induced nerve cell toxicity, changes in the Bax/caspase signaling pathway were analyzed. As revealed in Fig. 4A-E, overexpression of miR-323 significantly increases the ratio of $\mathrm{Bax} / \mathrm{Bcl}-2$, and increased caspase- 3 and caspase-9 activity levels in the in vivo model, compared with negative-control mimics group. By contrast, downregulation of miR-323 significantly suppressed the protein expression 

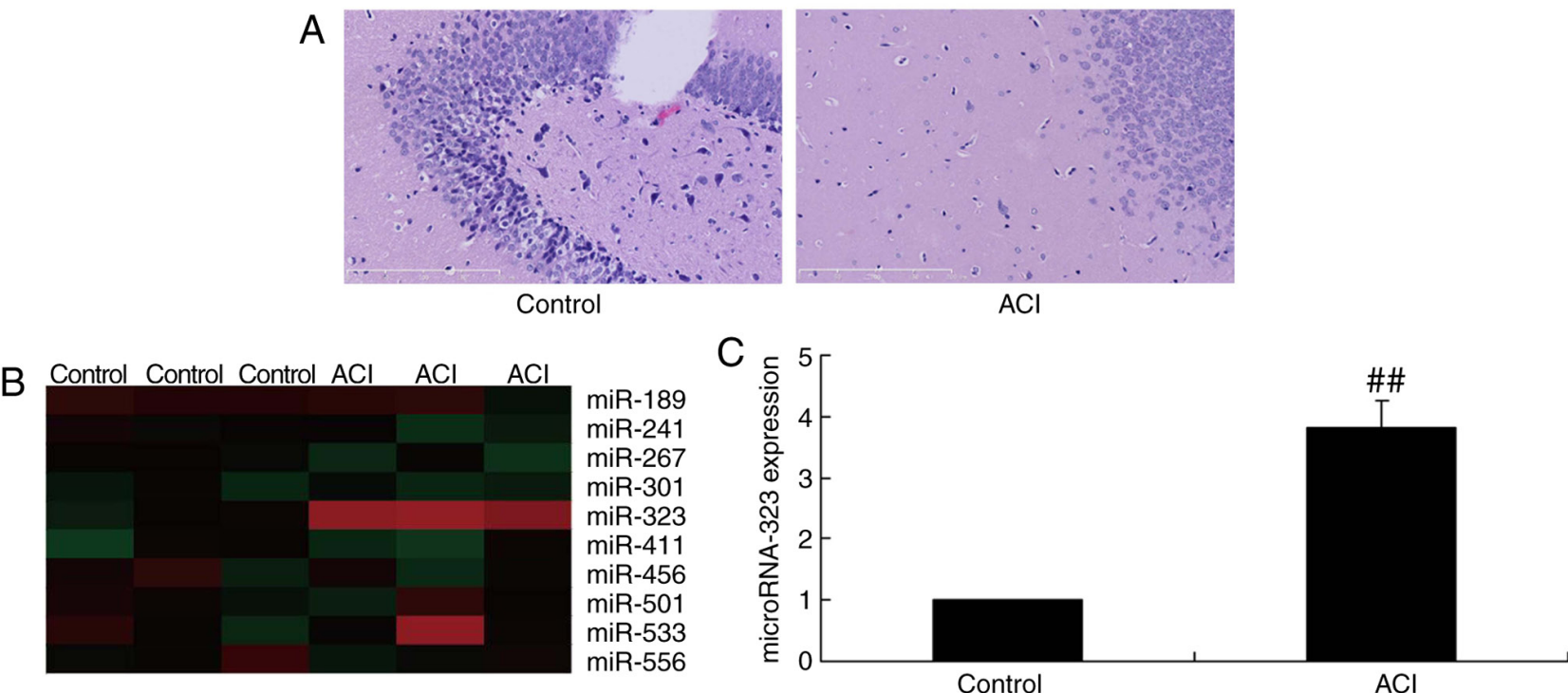

Figure 1. miR-323 expression in rats with cerebral infarction. (A) Hematoxylin and eosin staining of hippocampal neurons. (B) Gene chip analysis and (C) quantitative polymerase chain reaction for examination of miR-323 expression. ${ }^{\sharp \#} \mathrm{P}<0.01$ vs. control group. miR, microRNA; ACI, acute cerebral infarction.
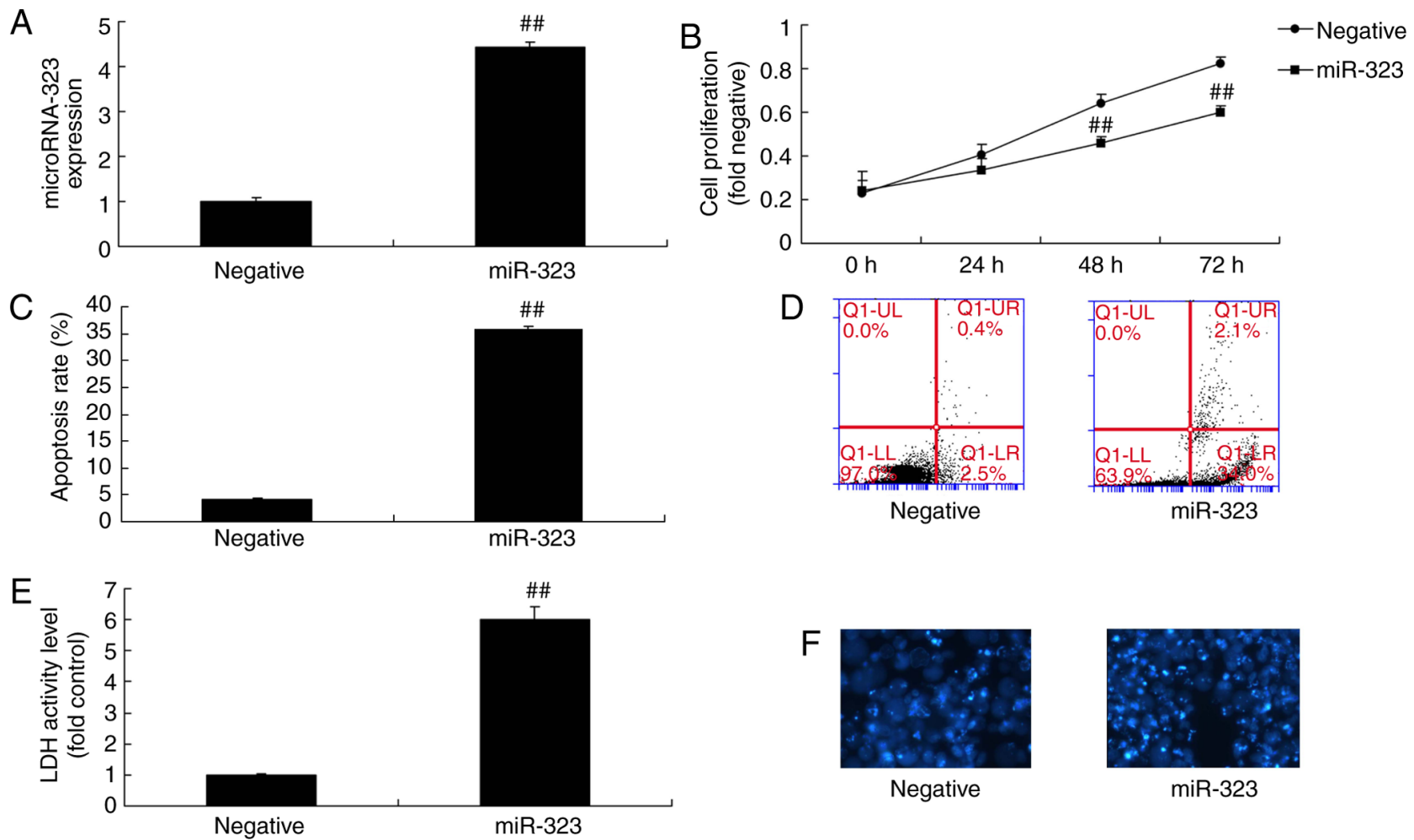

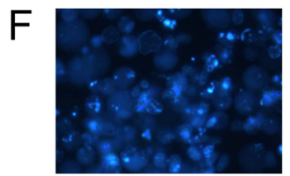

Negative

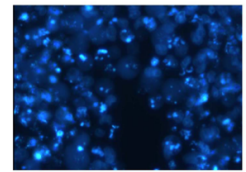

$\operatorname{miR}-323$

Figure 2. Overexpression of miR-323 regulates nerve cell toxicity in an in vitro model. (A) Quantitative polymerase chain reaction analysis of miR-323 expression. (B) Cell viability, (C) apoptosis rate, (D) flow cytometry results, (E) lactate dehydrogenase activity and (F) DAPI staining (magnification, $\mathrm{x} 200$ ). ${ }^{\# \#} \mathrm{P}<0.01$ vs. negative mimics group. miR, microRNA.

of the ratio of Bax/Bcl-2, and decreased caspease- 3 and caspase-9 activity levels in the in vivo model, compared with the negative-control mimics group (Fig. 4E-H). These results indicated that miR-323 regulated the Bax/caspase signaling pathway in the in vivo model.

miR-323 regulates TGF- $\beta 1 / S M A D 3$ signaling pathway in the in vivo model. Gene chip analysis was employed to analyze the signaling pathway changes in the in vivo model of ACI. The results revealed that SMAD3 was reduced upon overexpression of miR-323, compared with the negative control group (Fig. 5A). A dual-luciferase reporter assay was then performed to analyze the interaction of miR-323 and SMAD3. As shown in Fig. 5B, target sites were located in the 3'-UTR of SMAD3 mRNA. In addition, immunofluorescence analysis revealed that overexpression of miR-323 suppressed SMAD3 

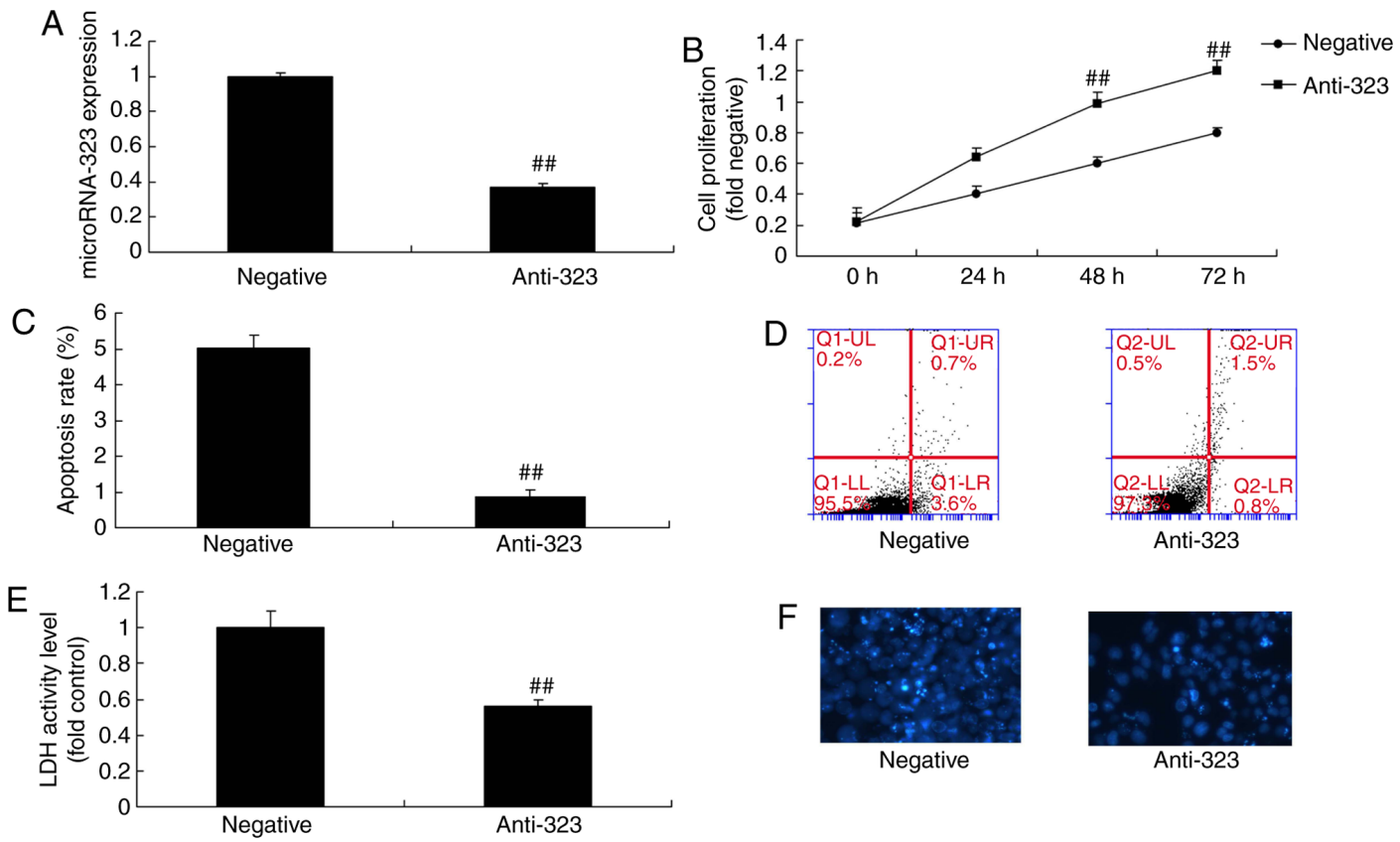

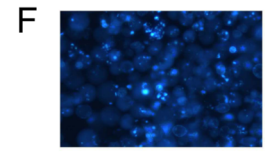

Negative

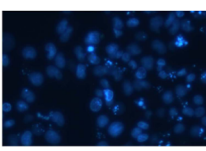

Anti-323

Figure 3. Downregulation of miR-323 regulates nerve cell toxicity in an in vitro model. (A) Quantitative polymerase chain reaction for miR-323 expression determination; (B) cell viability, (C) apoptosis rate, (D) flow cytometry, (E) DAPI staining (magnification, x200) and (F) lactate dehydrogenase activity. ${ }^{\# \#} \mathrm{P}<0.01$ vs. negative mimics group. miR, microRNA; $\mathrm{LDH}$, lactate dehydrogenase.
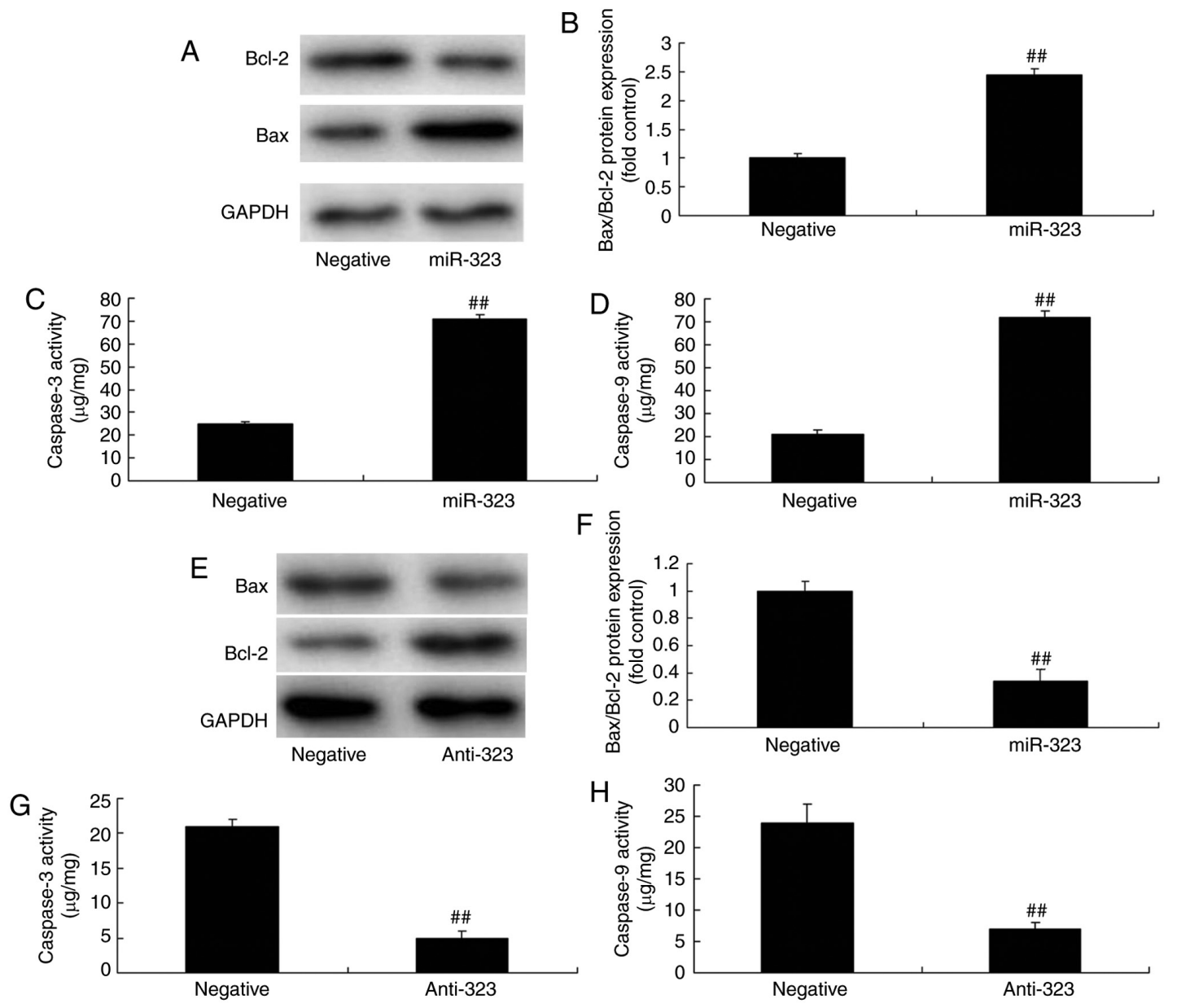

Figure 4. miR-323 regulates Bax/caspase signaling pathway in the in vitro model. (A) Bax and Bcl-2 protein expression were detected using western blot analysis, and (B) the quantified Bax/Bcl-2 expression is shown. (C) Caspase-3 and (D) caspase-9 activity levels following overexpression of miR-323. (E) Bax and $\mathrm{Bcl}-2$ protein expression detected by western blot analysis and $(\mathrm{F})$ the quantified $\mathrm{Bax} / \mathrm{Bcl}-2$ expression using statistical analysis. (G) Caspase-3 and (H) caspase-9 activity level determined by downregulation of miR-323. ${ }^{\# t} \mathrm{P}<0.01$ vs. negative mimics group. miR, microRNA; Bcl-2, B-cell lymphoma 2; Bax, Bcl-2-associated X protein. 
A Negative Negative Negative miR-323 miR-323 miR-323

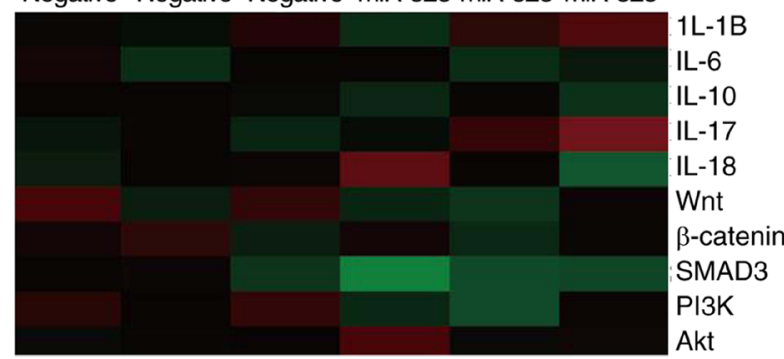

B microRNA-323 GCACAUUACAC

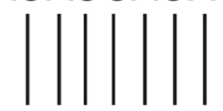

SMAD3 CAGGUAAUGUA
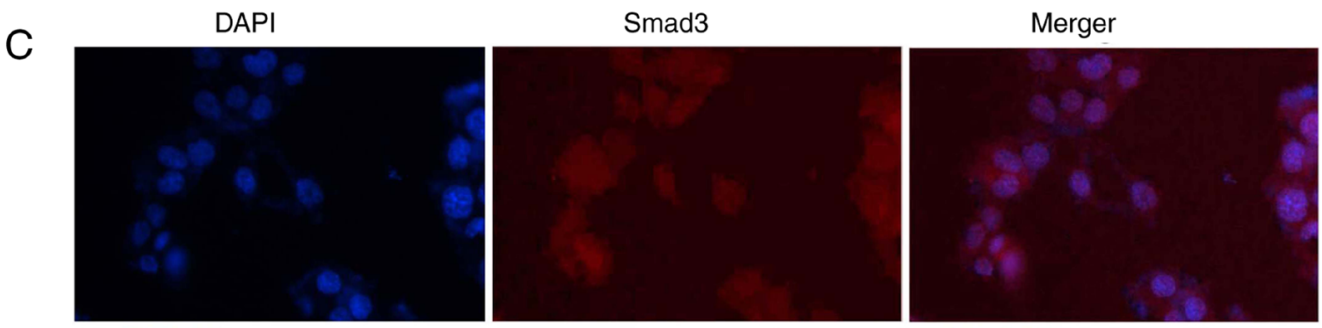

Control
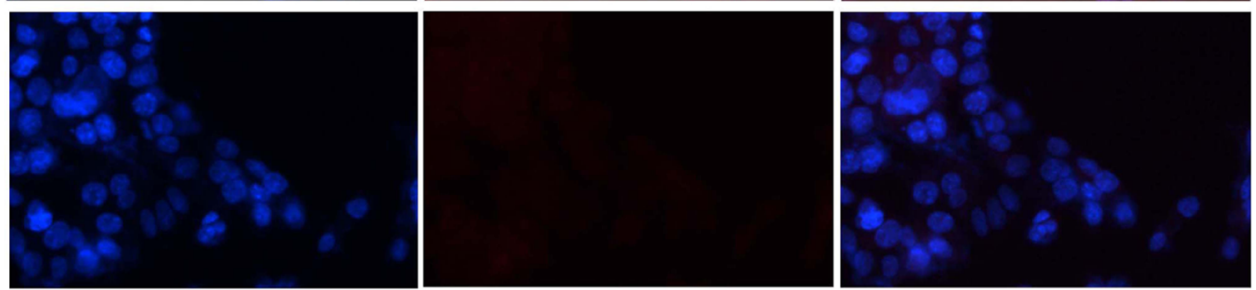

$\operatorname{miR}-323$

D

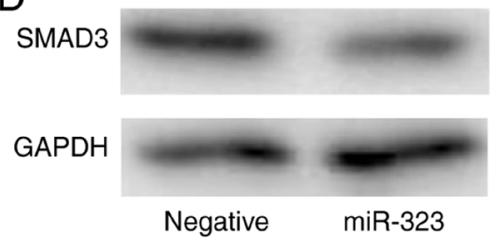

$\mathrm{F}$

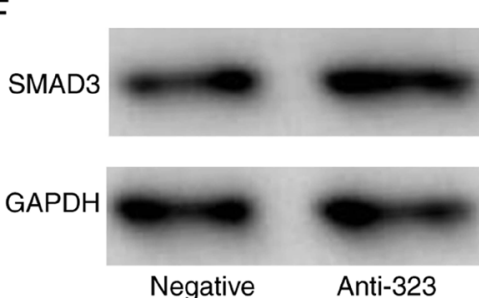

E

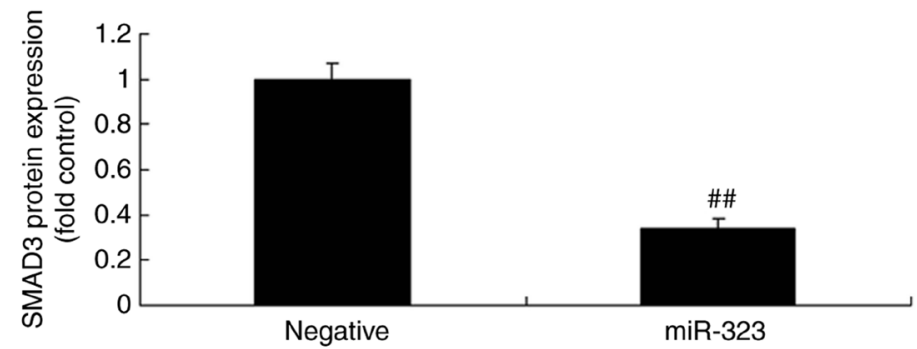

$\mathrm{G}$

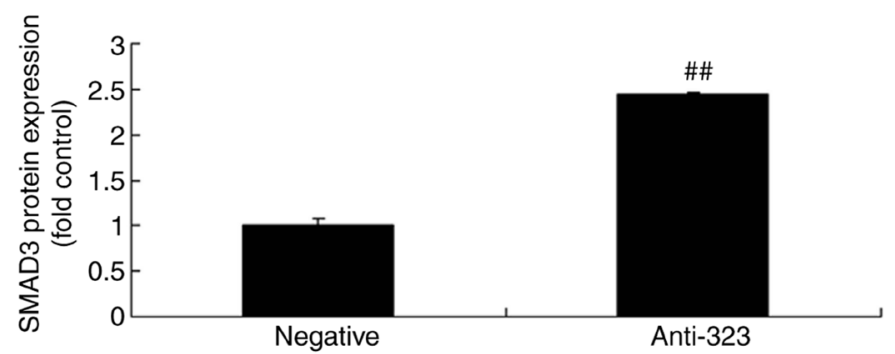

Figure 5. miR-323 regulates the TGF-31/SMAD3 signaling pathway in the in vitro model. (A) Gene chip analysis of the SMAD3 signaling pathway. (B) Partial sequence of the 3'-untranslated region of SMAD3 mRNA showing the predicted miR-323 target sites. (C) Immunofluorescence assay of SMAD3 protein expression (magnification, x200). (D) SMAD3 protein expression using western blot analysis and (E) quantified SMAD3 expression following overexpression of miR-323. (F) SMAD3 protein expression using western blot analysis and (G) quantified Bax/Bcl-2 expression following downregulation of miR-323. ${ }^{\# \#} \mathrm{P}<0.01$ vs. negative mimics group. miR, microRNA; TGF- $\beta 1$, transforming growth factor- $\beta 1$; Bcl-2, B-cell lymphoma 2 ; Bax, Bcl-2-associated X protein.

protein expression in the in vitro model of cerebral infarction, compared with that in the negative control group (Fig. 5C). Similarly, western blot analysis revealed that overexpression of miR-323 significantly suppressed SMAD3 protein expression in this cell model (Fig. 5D and E). By contrast, downregulation of miR-323 significantly induced SMAD3 protein expression in the in vitro model of cerebral infarction, compared with negative-control mimics group, as demonstrated by the 

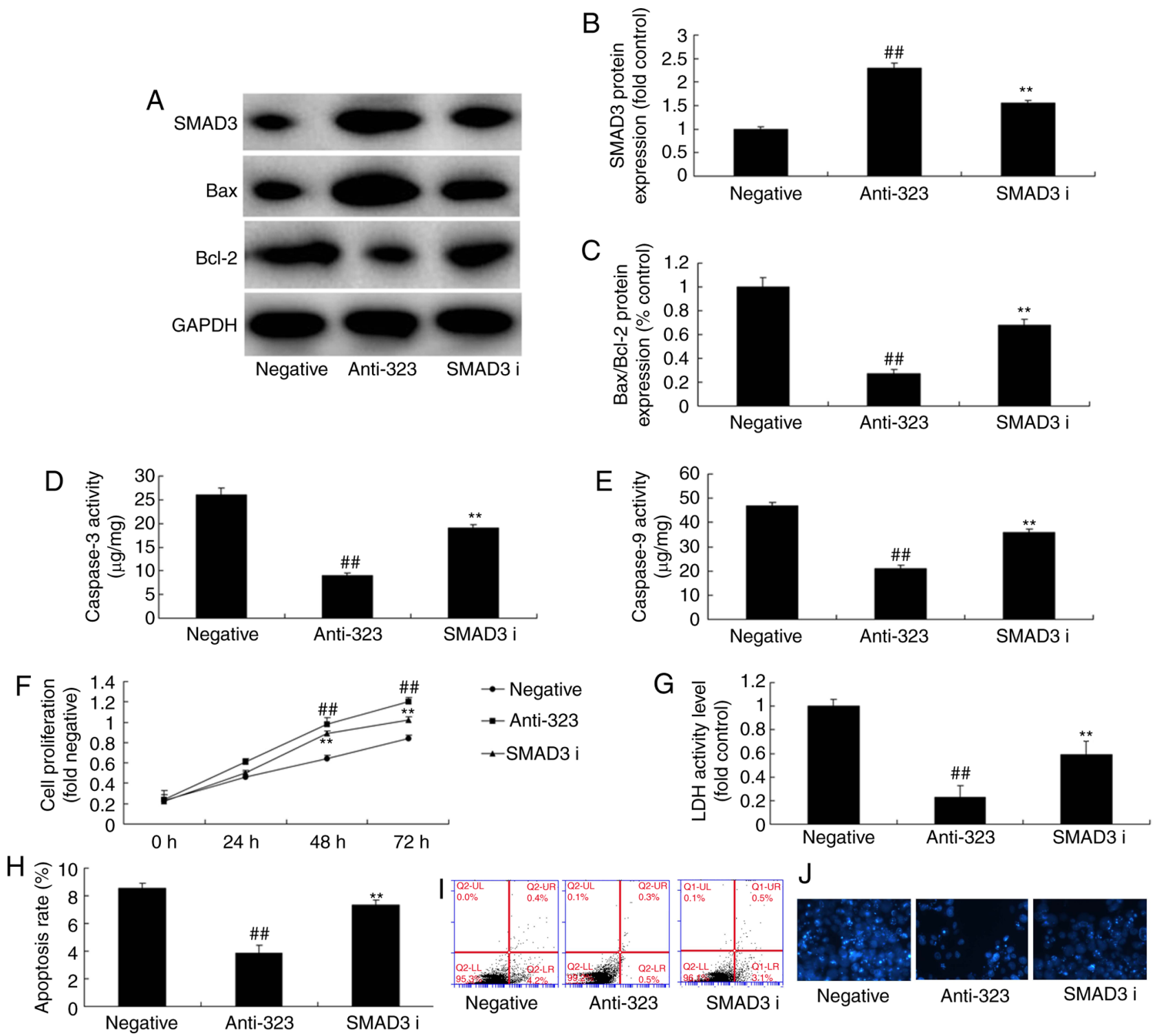

Figure 6. SMAD3 inhibitor participates in the effect of miR-323 in cerebral infarction through the transforming growth factor- $\beta 1 /$ SMAD3 signaling pathway. (A) Western blot analysis of SMAD3, Bax and Bcl-2 protein expression levels, and (B) SMAD3 and (C) Bax/Bcl-2 quantified expression levels; (D) caspase-3 and (E) caspase-9 activity levels; (F) cell proliferation; (G) LDH activity; (H) apoptosis rate; (I) flow cytometry results; and (J) DAPI staining (magnification, $\mathrm{x} 200) .{ }^{\# \#} \mathrm{P}<0.01$ vs. negative mimics group; ${ }^{* * *} \mathrm{P}<0.01$ vs. anti-miR-323 group. SMAD3 i, downregulation of miR-323 and SMAD3 inhibitor group; miR, microRNA; Bcl-2, B-cell lymphoma 2; Bax, Bcl-2-associated X protein.

immunoassay results (Fig. 5F and G). These results revealed that miR-323 regulates the TGF- $\beta 1 /$ SMAD3 signaling pathway, which suppressed nerve cell toxicity in cerebral infarction.

SMAD3 inhibitor participates in the effect of miR-323 in cerebral infarction through the TGF- $\beta 1 / S M A D 3$ signaling pathway. To further explore the function of SMAD3 in the effect of miR-323 in cerebral infarction, a SMAD3 inhibitor (SIS3) was used in the in vitro model. It was observed that SMAD3 inhibitor significantly suppressed SMAD3 protein expression, induced $\mathrm{Bax} / \mathrm{Bcl}-2$ protein expression and increased caspase-3/caspase-9 activity levels in in vitro model of cerebral infarction following downregulation miR-323, compared with the miR-323 downregulation group (Fig. 6A-E). In addition, the SMAD3 inhibitor in combination with miR-323 downregulation reduced cell viability and LDH activity level in the in vitro model, compared with the downregulation miR-323 group (Fig. 6E-J). These results demonstrated that SMAD3 is associated with the effects of miR-323 in cerebral infarction.

TGF- $\beta 1$ inhibitor participates in the effect of miR-323 in cerebral infarction through the TGF- $\beta 1 / S M A D 3$ signaling pathway. To examine whether the TGF- $\beta 1 /$ SMAD3 signaling pathway is involved in the effect of miR-323 in cerebral infarction, TGF- $\beta 1$ inhibitor was utilized to suppress the TGF- $\beta 1 /$ SMAD3 signaling pathway. As a result, TGF- $\beta 1$ inhibitor, $20 \mathrm{~nm}$ of LY2109761, significantly suppressed SMAD3 protein expression, induced $\mathrm{Bax} / \mathrm{Bcl}-2$ protein expression, and increased caspase-3/caspase-9 activity levels in the in vitro model of cerebral infarction following the downregulation of miR-323, as compared with the anti-miR-323 group (Fig. 7A-F). Meanwhile, TGF- $\beta 1$ inhibitor in combination with anti-miR-323 significantly reduced the effect of miR-323 downregulation on cell viability and LDH 

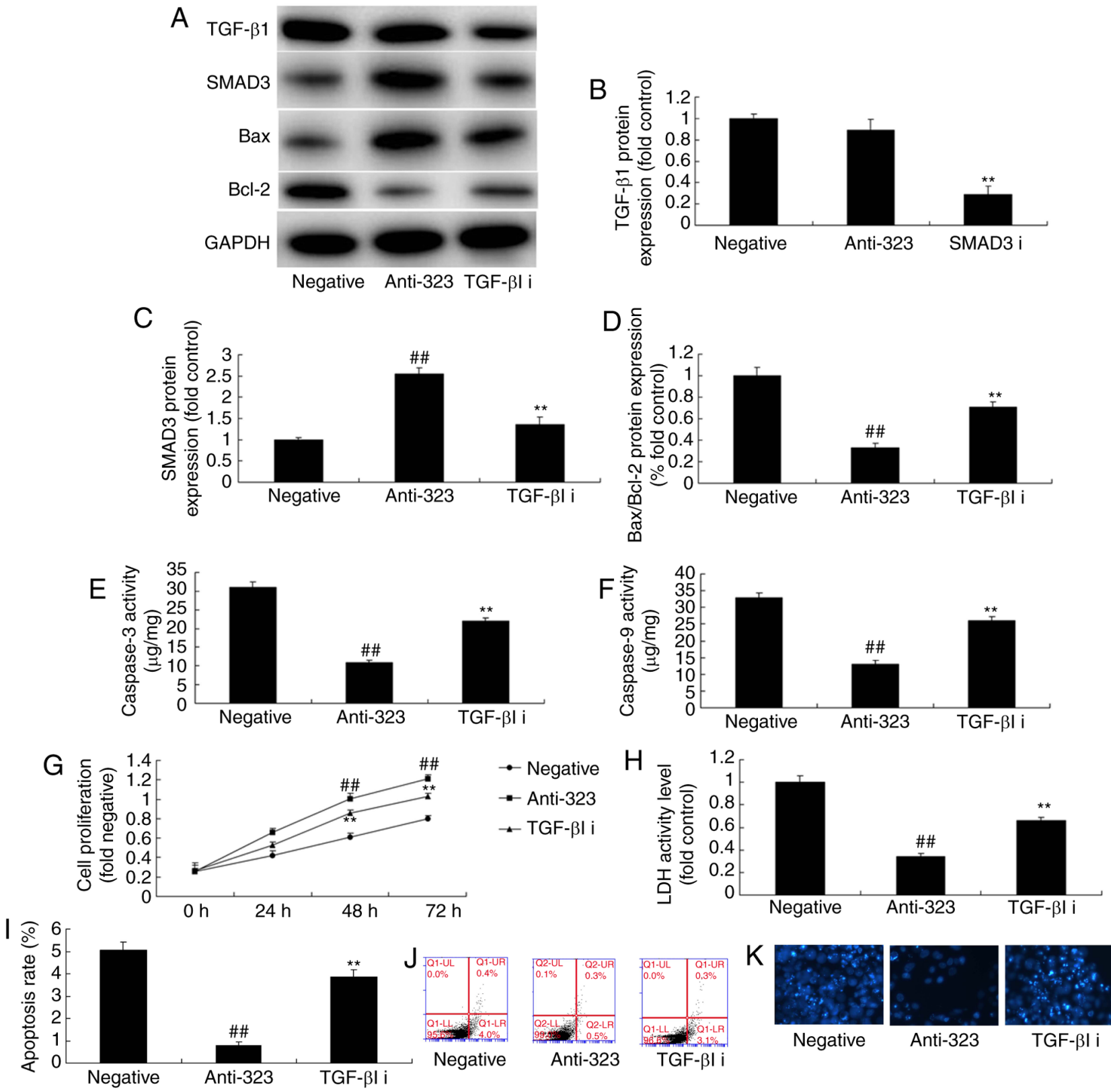

Figure 7. TGF- $\beta 1$ inhibitor participates in the effect of miR-323 in cerebral infarction through the TGF- $\beta 1 /$ SMAD3 signaling pathway. (A) Western blot analysis of TGF- $\beta 1$, SMAD3, Bax and Bcl-2 protein expression, and quantified levels of (B) TGF- $\beta 1$, (C) SMAD3 and (D) Bax/Bcl-2. (E) Caspase-3 and (F) caspase-9 activity levels. (G) Cell proliferation rate. (H) LDH activity. (I) Apoptosis rate and (J) flow cytometry results. (K) DAPI staining. ${ }^{\# \# P} \mathrm{P}<0.01 \mathrm{vs}$. negative mimics group; ${ }^{* *} \mathrm{P}<0.01$ vs. anti-miR-323 group. TGF- $\beta 1$, transforming growth factor- $\beta 1 ;$ miR, microRNA; TGF- $\beta 1$ i, downregulation of miR-323 and TGF- $\beta 1$ inhibitor group; Bcl-2, B-cell lymphoma 2; Bax, Bcl-2-associated X protein.

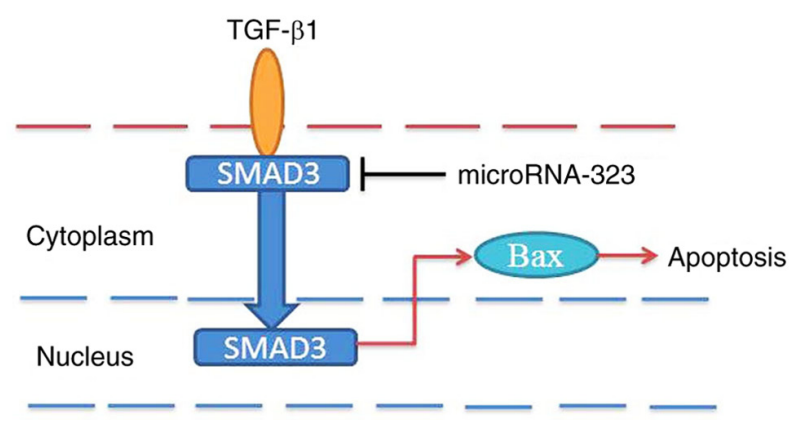

Figure 8. MicroRNA-323 suppresses nerve cell toxicity in cerebral infarction via the TGF- $31 /$ SMAD3 signaling pathway. MicroRNA-323 suppressed SMAD3/Bax protein expression, which decreased nerve cell toxicity and apoptosis rates in cerebral infarction. TGF- $\beta 1$, transforming growth factor- $\beta 1$. activity level in the in vitro model when compared with anti-miR-323 group (Fig. 7G-K). These results suggested that the miR-323/TGF- $\beta 1 /$ SMAD3 signaling pathway regulated cell death during cerebral infarction.

\section{Discussion}

$\mathrm{ACI}$, also referred to as ischemic stroke, is a pathological process of neural dysfunction or injury within brain tissue that results from local cerebral ischemia and dysfunction (9). The morbidity and mortality of ACI patients is increasing annually in clinical practice (2). This can be attributed to improvement in diagnostic technologies (9). The data of the present study demonstrated that miR-323 expression was upregulated in rats 
with cerebral infarction as compared that in the sham-control group. Yang et al (10) indicated that miR-323 regulated ischemia/reperfusion-induced rat neuronal cell death. The current study only used immunohistochemical analysis to assess the effects of miR-323 on SMAD3 in the in vivo model; however, this is an insufficient, and future studies should use in situ hybridization histochemical and immunohistochemical analyses to analyze the function of miR-323 in ACI.

Certain miRNAs serve important roles in neurogenesis and neuronal function, even at low concentrations. The majority of miRNAs are located in the tissues and cells (11), while they can also stably exist in body fluids, including blood, urine and amniotic fluid (12). Human tissue miRNA detection can hardly be achieved or repeatedly used in clinical practice. A previous study reported that there are stable miRNA molecules in the peripheral blood (4). Furthermore, different miRNA expression profiles are identified in different diseases (12). These discoveries have initiated the novel strategy of using peripheral blood miRNAs for non-invasive disease diagnosis (4). Research reported that peripheral blood miRNAs with specific expression changes can be found in multiple diseases, including tumor, diabetes, myocardial infarction, Parkinson' disease and Alzheimer's disease (11). In the present study, overexpression of miR-323 was found to significantly increase LDH activity level and inhibit cell viability in an in vitro model of ACI. These results suggested that miR-323 induced nerve cell apoptosis in ACI.

TGF- $\beta$ exerts multiple effects, such as anti-oxidation, blocking apoptosis, regulating the inflammatory response, and regulating microglial cell and astrocyte responses (13). It can also inhibit central nervous system inflammatory response at the early stage of ischemia (13), therefore alleviating encephaledema, reducing the infarct size and promoting microangiogenesis (14). Besides, it serves an important role in the repair of brain tissue injury. A study on a rat ischemia and hypoxia model verified that exogenous TGF- $\beta$ alleviated the microglial cell response and reduced neuron death, as well as the infarct size (14). In the current study, overexpression of miR-323 was found to significantly suppress SMAD3 protein expression in an in vitro model of cerebral infarction. Wang et al (15) suggested that miR-323-3p inhibited cell invasion and metastasis in pancreatic ductal adenocarcinoma via SMAD2 and SMAD3.

TGF- $\beta$ cytokine is a multi-functional protein that is closely associated with cell proliferation, differentiation, apoptosis and angiogenesis (16). In the case of injury, the nonparenchymal cells release TGF- $\beta$, which then binds with the cell surface receptor and promotes the phosphorylation of other TGF- $\beta$ receptors through a positive feedback (16). TGF- $\beta$ can also activate the Smad pathway. The phosphorylated TGF- $\beta$ receptor renders spatial conformational changes in the intracellular nucleus-cytoplasm signaling molecules SMAD2 and SMAD3 through phosphorylation $(17,18)$. Thereby, these molecules cross-link with Smad4 to form an isotrimer complex, which subsequently enters the nucleus to regulate target gene transcription (18). Finally, it will promote oxidative stress, inflammatory response, fibrosis and apoptosis $(18,19)$. In the current study, it was demonstrated that the TGF- $\beta 1$ inhibitor participated in the effect of miR-323 in cerebral infarction through the TGF- $\beta 1 /$ SMAD3 signaling pathway. Kärner et al (20) further suggested that increased miR-323-3p in asthma served a role in the regulation of the TGF- $\beta$ pathway and interleukin-22 production. These results were in agreement with the findings of present study, indicating that miR-323 suppresses nerve cell toxicity in cerebral infarction through the TGF- $\beta 1 /$ SMAD3 signaling pathway.

In conclusion, the current data demonstrated for the first time, to the best of our knowledge, that miR-323 suppresses nerve cell apoptosis in cerebral infarction via the TGF- $\beta 1 /$ SMAD3 signaling pathway (Fig. 8). The study expanded on the understanding of the pathogenic role of miR-323 in cerebral infarction via TGF- $\beta 1 /$ SMAD3 signaling pathway, and this miRNA may represent a crucial target for potential therapeutic intervention.

\section{Acknowledgements}

Not applicable.

\section{Funding}

No funding was received.

\section{Availability of data and materials}

The analyzed data sets generated during the present study are available from the corresponding author on reasonable request.

\section{Authors' contributions}

FC designed the present study. HD, JW, WZ, ZC and YT performed the experiments. FC and HD analyzed the data. FC wrote the manuscript. All authors read and approved the final manuscript.

\section{Ethics approval and consent to participate}

Animal experiments were approved by the Ethics Committee of the Beijing Luhe Hospital Capital Medical University (Beijing, China).

\section{Patient consent for publication}

Not applicable.

\section{Competing interests}

The authors declare that they have no competing interests.

\section{References}

1. Al-Ali F, Berkhemer OA, Yousman WP, Elias JJ, Bender EN, Lingsma HF, van der Lugt A, Dippel DW, Roos YB, van Oostenbrugge RJ, et al: The capillary index score as a marker of viable cerebral tissue: Proof of concept-the capillary index score in the MR CLEAN (multicenter randomized clinical trial of endovascular treatment for acute ischemic stroke in the Netherlands) trial. Stroke 47: 2286-2291, 2016.

2. Maeshima S, Okamoto S, Okazaki H, Mizuno S, Asano N, Tsunoda T, Maeda H, Masaki M and Sonoda S: Hemorrhagic transformation in patients with cerebral infarction referred to a rehabilitation hospital. Interv Neurol 4: 69-74, 2016.

3. Kim DH, Kim SU, Sung JH, Lee DH, Yi HJ and Lee SW: Significances and outcomes of mechanical thrombectomy for acute infarction in very elderly patients: A single center experience. J Korean Neurosurg Soc 60: 654-660, 2017. 
4. Fasanaro P, Greco S, Ivan M, Capogrossi MC and Martelli F: microRNA: Emerging therapeutic targets in acute ischemic diseases. Pharmacol Ther 125: 92-104, 2010.

5. Khanna S, Rink C, Ghoorkhanian R, Gnyawali S, Heigel M, Wijesinghe DS, Chalfant CE, Chan YC, Banerjee J, Huang Y, et al: Loss of miR-29b following acute ischemic stroke contributes to neural cell death and infarct size. J Cereb Blood Flow Metab 33: 1197-1206, 2013.

6. Kim JM, Jung KH, Chu K, Lee ST, Ban J, Moon J, Kim M, Lee SK and Roh JK: Atherosclerosis-related circulating MicroRNAs as a predictor of stroke recurrence. Transl Stroke Res 6: 191-197, 2015.

7. Selvamani A, Williams MH, Miranda RC and Sohrabji F: Circulating miRNA profiles provide a biomarker for severity of stroke outcomes associated with age and sex in a rat model. Clin Sci (Lond) 127: 77-89, 2014.

8. Slevin M, Krupinski J, Slowik A, Kumar P, Szczudlik A and Gaffney J: Serial measurement of vascular endothelial growth factor and transforming growth factor-betal in serum of patients with acute ischemic stroke. Stroke 31: 1863-1870, 2000

9. Dowling MM, Quinn CT, Plumb P, Rogers ZR, Rollins NK Koral K and Buchanan GR: Acute silent cerebral ischemia and infarction during acute anemia in children with and without sickle cell disease. Blood 120: 3891-3897, 2012.

10. Yang L, Xiong Y, Hu XF and Du YH: MicroRNA-323 regulates ischemia/reperfusion injury-induced neuronal cell death by targeting BRI3. Int J Clin Exp Pathol 8: 10725-10733, 2015.

11. Dewdney B, Trollope A, Moxon J, Thomas Manapurathe D, Biros E and Golledge J: Circulating MicroRNAs as biomarkers for acute ischemic stroke: A Systematic review. J Stroke Cerebrovasc Dis 27: 522-530, 2018.

12. Zhou J and Zhang J: Identification of miRNA-21 and miRNA-24 in plasma as potential early stage markers of acute cerebral infarction. Mol Med Rep 10: 971-976, 2014.

13. Kim JS, Yoon SS, Kim YH and Ryu JS: Serial measurement of interleukin-6, transforming growth factor-beta, and S-100 protein in patients with acute stroke. Stroke 27: 1553-1557, 1996.
14. Ata KA, Lennmyr F, Funa K, Olsson Y and Terent A: Expression of transforming growth factor-beta1, 2, 3 isoforms and type I and II receptors in acute focal cerebral ischemia: An immunohistochemical study in rat after transient and permanent occlusion of middle cerebral artery. Acta Neuropathol 97: 447-455, 1999.

15. Wang C, Liu P, Wu H, Cui P, Li Y, Liu Y, Liu Z and Gou S: MicroRNA-323-3p inhibits cell invasion and metastasis in pancreatic ductal adenocarcinoma via direct suppression of SMAD2 and SMAD3. Oncotarget 7: 14912-14924, 2016.

16. Ronaldson PT, Demarco KM, Sanchez-Covarrubias L, Solinsky CM and Davis TP: Transforming growth factor-beta signaling alters substrate permeability and tight junction protein expression at the blood-brain barrier during inflammatory pain. J Cereb Blood Flow Metab 29: 1084-1098, 2009.

17. Lan W, Yang F, Li Z, Liu L, Sang H, Jiang Y, Xiong Y and Zhang R: Human urine kininogenase attenuates balloon-induced intimal hyperplasia in rabbit carotid artery through transforming growth factor $\beta 1 /$ SMAD2/3 signaling pathway. J Vasc Surg 64: 1074-1083, 2016

18. Tinoco-Veras CM, Santos AAQA, Stipursky J, Meloni M, Araujo APB, Foschetti DA, López-Ureña D, Quesada-Gómez C, Leitão RFC, Gomes FCA and Brito GAC: Transforming growth factor $\beta 1 / \mathrm{SMAD}$ signaling pathway activation protects the intestinal epithelium from clostridium difficile toxin a-induced damage. Infect Immun 85: e00430-17, 2017.

19. Grand Moursel L, Munting LP, van der Graaf LM, van Duinen SG, Goumans MTH, Ueberham U, Natté R, van Buchem MA, van Roon-Mom WMC and van der Weerd L: TGF $\beta$ pathway deregulation and abnormal phospho-SMAD2/3 staining in hereditary cerebral hemorrhage with amyloidosis-Dutch type. Brain Pathol 28: 495-506, 2018.

20. Kärner J, Wawrzyniak M, Tankov S, Runnel T, Aints A, Kisand K, Altraja A, Kingo K, Akdis CA, Akdis M and Rebane A: Increased microRNA-323-3p in IL-22/IL-17-producing T cells and asthma: A role in the regulation of the TGF- $\beta$ pathway and IL-22 production. Allergy 72: 55-65, 2017. 